\title{
A Note of Appreciation
}

The journal's mission (the psychosomatic home of innovative and integrative ideas in behavioral and medical sciences) could not be pursued without the help of the external referees, who have supplemented the editorial board in reviewing the manuscripts submitted to Psychotherapy and Psychosomatics.
Both external referees and editorial board members have disclosed potential conflicts of interest. The editorin-chief has no conflict of interest to declare for 2009. We would like to thank also Carlotta Belaise, who has taken care of press releases. The journal's impact factor (4.21 in 2008) confirms its growing influence in the literature.
A. Abbass (Halifax, N.S., Canada)

G. Abbate-Daga (Turin, Italy)

B.B. Arnetz (Detroit, Mich., USA)

A.S. Bahrick (Iowa City, Iowa, USA)

J.P. Barber (Philadelphia, Pa., USA)

A. Barbosa (Lisboa, Portugal)

A. Bartak (Halsteren, The Netherlands)

M. Battaglia (Milan, Italy)

B. Baune (Townsville, Qld., Australia)

C. Belaise (Bologna, Italy)

S. Bellingrath (Bremen, Germany)

R.H. Belmaker (Beer Sheva, Israel)

F. Benazzi (Forlì, Italy)

D. Benninghoven (Lübeck, Germany)

M. Beresnevaite (Kaunas, Lithuania)

C.L.H. Bockting (Groningen,

The Netherlands)

N. Bodde (Heeze, The Netherlands)

T.K. Bouman (Groningen,

The Netherlands)

S. Büchi (Zurich, Switzerland)

E. Bui (Toulouse, France)

A. Buske-Kirschbaum (Dresden, Germany)

G. Carrà (Milan, Italy)

B. Carroll (Carmel, Calif., USA)

M. Chiesa (Richmond, UK)

D. Cohen (Paris, France)

B. Colagiuri (Sydney, N.S.W., Australia)

F. Cosci (Firenze, Italy)

J.A. Cottraux (Lyon, France)

K. Davidson (New York, N.Y., USA)

M. Davis (Phoenix, Ariz., USA)
J. de Figuereido (Cheshire, Conn., USA)

J. De Fruyt (Brugge, Belgium)

A. delle Fave (Milan, Italy)

N. Delsedime (Torino, Italy)

U. Ehlert (Zurich, Switzerland)

S. Fabbri (Charlottesville, Va., USA)

F. Facchinetti (Modena, Italy)

A. Favaro (Padova, Italy)

E. Ferguson (Nottingham, UK)

S. Ferrari (Modena, Italy)

A. Finset (Oslo, Norway)

I. Franken (Rotterdam, The Netherlands)

M. Fullana (London, UK)

P. Fusar Poli (London, UK)

S. Galderisi (Napoli, Italy)

S.N. Ghaemi (Boston, Mass., USA)

A.T. Gloster (Dresden, Germany)

J. Goethe (Hartford, Conn., USA)

C. Gold (Bergen, Norway)

H.J. Grabe (Stralsund, Germany)

L. Grassi (Ferrara, Italy)

N. Greenberg (London, UK)

C. Grilo (New Haven, Conn., USA)

J. Guidi (Bologna, Italy)

A. Guolo (Verona, Italy)

M. Haerter (Freiburg, Germany)

D. Healy (Bangor, UK)

P. Hoglend (Oslo, Norway)

K. Honkalampi (Kuopio, Finland)

J. Hoyer (Dresden, Germany)

R.B. Jarrett (Dallas, Tex., USA)

L. Jaycox (Pittsburgh, Pa., USA)

E. Joosten (Nijmegen, The Netherlands)
R.S. Jorgensen (Syracuse, N.Y., USA)

R.A. Kanaan (London, UK)

G. Keitner (Providence, R.I., USA)

D. Kemp (Cleveland, Ohio, USA)

I. Kirsch (Hull, UK)

J. Knoop (Nijmegen, The Netherlands)

W. Kuyken (Exeter, UK)

C. Lahmann (Munich, Germany)

C. Lai (Rome, Italy)

J. Leckman (New Haven, Conn., USA)

P. Leombruni (Torino, Italy)

S. Levenstein (Rome, Italy)

S. Lilienfeld (Atlanta, Ga., USA)

D. Linder (Venice, Italy)

M. Linehan (Seattle, Wash., USA)

A. Lobo (Zaragoza, Spain)

M.A. Lumley (Detroit, Mich., USA)

T. Lundin (Uppsala, Sweden)

G. Majani (Pavia, Italy)

M. Menchetti (Bologna, Italy)

B. Metternich (Freiburg, Germany)

T. Meyer (Marburg, Germany)

N. Micali (London, UK)

R. Mota Cardoso (Porto, Portugal)

S. Munsch (Basel, Switzerland)

N. Navarrete (Granada, Spain)

K. Nicholson Perry (Sydney, N.S.W., Australia)

A.A. Nierenberg (Boston, Mass., USA)

G. Northoff (Magdeburg, Germany)

H. Ochitill (San Francisco, Calif., USA)

E. Offidani (Bologna, Italy)

\section{KARGER}

Fax +41 613061234 E-Mail karger@karger.ch www.karger.com
(C) 2009 S. Karger AG, Basel

0033-3190/10/0791-0004\$26.00/0 
J.S. Ogrodniczuk (Vancouver, B.C., Canada)

F. Ottolini (Bologna, Italy)

M. Pasquini (Rome, Italy)

S. Patten (Calgary, Alta., Canada)

P. Pedrelli (Boston, Mass., USA)

C. Peretti (Paris, France)

R.H. Perlis (Boston, Mass., USA)

A. Picardi (Rome, Italy)

P. Porcelli (Bari, Italy)

M. Powers (Philadelphia, Pa., USA)

A. Preti (Cagliari, Italy)

L.C. Quilty (Toronto, Ont., Canada)

G. Ranjith (Beckenham, UK)

V. Ricca (Firenze, Italy)

M. Rigatelli (Modena, Italy)

Z. Rihmer (Budapest, Hungary)

B.R. Rutherford (New York, N.Y., USA)
J.K. Salminen (Turku, Finland)

M. Schredl (Mannheim, Germany)

I.E. Schulte (Bremen, Germany)

S. Shiri (Tel Aviv, Israel)

L. Sirri (Bologna, Italy)

N. Sonino (Padova, Italy)

G.I. Spielmans (St. Paul, Minn., USA)

C. Spitzer (Stralsund, Germany)

L. Staccini (Bologna, Italy)

F. Stiefel (Lausanne, Switzerland)

A. Sumathipala (London, UK)

J. Svaldi (Freiburg, Germany)

N. Tarrier (Manchester, UK)

G.J. Taylor (Toronto, Ont., Canada)

O. Todarello (Bari, Italy)

R.A. Topciu (Rochester, N.Y., USA)

E. Tossani (Bologna, Italy)

H.L. Van (Amsterdam, The Netherlands)
J.H. van der Meulen (London, UK)

S. Vanheule (Gent, Belgium)

B. Van Houdenhove (Leuven, Belgium)

J.P. van Melle (Groningen,

The Netherlands)

D. Visani (Bologna, Italy)

H. Walach (Northampton, UK)

F. Wilhelm (Basel, Switzerland)

V. Wittchen (Dresden, Germany)

L. Wittmann (Zurich, Switzerland)

B. Wood (Buffalo, N.Y., USA)

J. Wright (Louisville, Ky., USA)

W. Yeung (Phoenix, Ariz., USA)

C. Zimmermann (Verona, Italy)

S. Zipfel (Tübingen, Germany)

D. Zolnierczyk-Zreda (Warsaw, Poland) 